\title{
ON THE EXPLOITATION OF COLLABORATIVE ARGUMENTATION STRUCTURES FOR INDUCING REASONING BEHAVIOR
}

\author{
Nikos Karacapilidis ${ }^{1}$ and Vassilis Tampakas ${ }^{2}$ \\ ${ }^{I}$ IMIS Lab, MEAD, University of Patras, 26504 Rio Patras, Greece \\ ${ }^{2}$ Electrical and Computer Engineering Department, University of Peloponnese, Greece
}

\begin{abstract}
Contemporary collaborative argumentation support systems provide limited or no support in analyzing and inducing their users' reasoning behavior. This article discusses the empowerment of such systems towards this direction through an innovative approach, which is based on the assessment of similar argumentation sequences. Representative cases where the proposed approach may be used to capture the users' behavior, improve their collaboration and increase the argumentation support system's awareness are examined.
\end{abstract}

\section{KEYWORDS}

Argumentation, Decision Making, Reasoning, Collaboration, IBIS

\section{INTRODUCTION}

Argumentation has been proven to be a very efficient process in cases where people attempt to resolve ill-defined problems and conflicting scenarios. The study of argumentation dates long before the "information age" and constitutes a cross-disciplinary research field. Scientists from different backgrounds such as linguistics, philosophy, education and recently from informatics and computer science have been actively involved in argumentation theory and related software systems.

Argumentation support systems are built on top of alternative argumentation models. Although each model serves a specific purpose, they all share some common characteristics: they define the types of argumentation objects and the actions that can be performed on them. Thus, once the system has adopted a model, every discourse is codified through and validated against it. Contemporary argumentation support systems usually impose sophisticated argumentation structures. However, they offer limited or no support in the analysis of the argumentation structure; they monitor the users' contributions in a passive way; provide limited feedback about the focus of the discussion and fail to assess or predict the evolution of an argumentative discourse.

As revealed from the argumentation related literature, individuals react to and understand the same input in different ways. For instance, people may overlook information that undermines their positions (the confirmation bias phenomenon (Jonas et al., 2001)) and show preference to supportive, rather than opposing (the selective exposure phenomenon (Kuhn, 1991)). Furthermore, newcomers have to face even more challenges, such as the examination of specific parts of a dialogue, the reconstruction of the discourse, and the identification of inconsistencies. Additionally, community leaders are facing the problem of keeping an overview of the developing discussions in order to intervene and retain the desired discourse objectives. For instance, there are cases where an argument might raise a new debate over a certain claim and users might be swept off to minor or irrelevant issues. We argue that such concerns are of major importance for maintaining a high quality argumentative discourse and that there appears to be limited system support with regards to these.

Motivated by similar initiatives that aim at organizing and understanding the enormous amount of user generated data, work reported in this paper focuses on the exploitation of argumentation structures for automatically inducing users' behavior in an argumentation context. The proposed approach enables the 
analysis of users' contributions in argumentation support systems and is based on the assessment of similar argumentation sequences.

The remainder of the paper is structured as follows. Section 2 provides a short introduction to the basic concepts and the history of argumentation support systems. Section 3 describes the mechanics of our approach in an intuitive and simple way. Section 4 presents and discusses representative examples about how this approach may be found useful. The last section concludes with some final remarks.

\section{ARGUMENTATION SUPPORT SYSTEMS}

The IBIS (Issue Based Information System (Kunz and Rittel, 1970)) has been one of the most influential systems that supports argumentation and group decision support. According to the IBIS approach, information units are classified as issues, positions or arguments. Users participating in a discussion can comment about problems or claims that are reflected in the corresponding visualized logical diagram. The aim of this activity is to support collective understanding and team-based reasoning. As a specific example, consider the instance of an argumentative discussion following the IBIS formalization shown in Figure 1. In this example, Reason!Able is used to chart an argumentative discussion taking place among several participants (van Gelder, 2003). Numerous arguments are proposed and linked to each other. The argumentation tree shown may constitute a refined, small part of the actual deliberation.

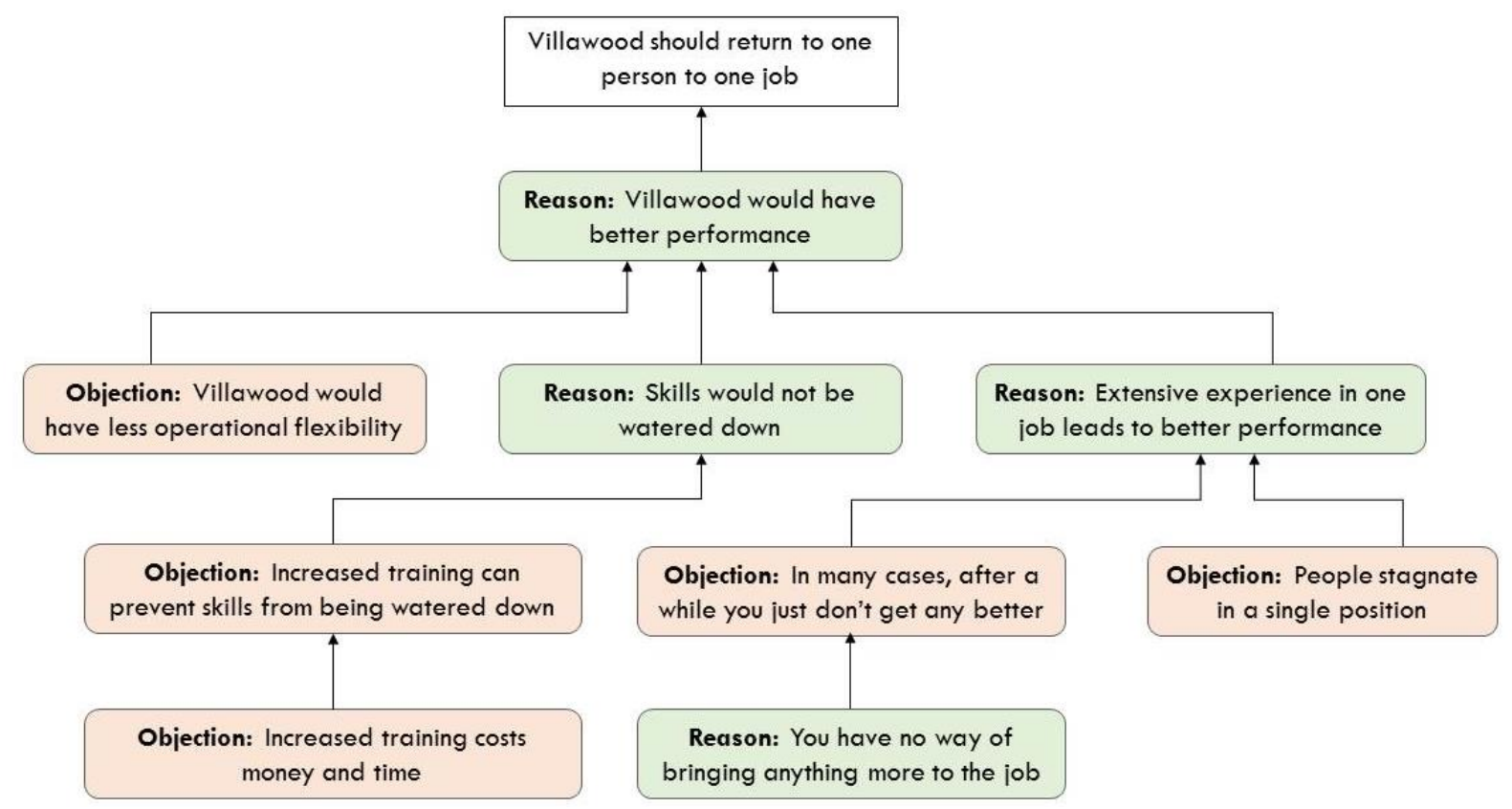

Figure 1. An instance of a partial argumentation tree, as visualized in Reason!Able (taken from (van Gelder, 2003)). The top node represents one conclusion (or claim) concerning the factory named Villawood, while the rest of the nodes are arguments clustered and linked to each other through support or opposition

A comprehensive review of existing argumentation support systems exceeds the scope of this paper. However, a short discussion about the evolution of the argumentation support systems follows. As shown in Table 1, systems that belong to the first generation were developed in the realms of hypertext systems. For instance, gIBIS (Conklin and Begeman, 1987) allow the visualization and interconnection of arguments. Nevertheless, the information units supported are mainly documented and no constraint imposed by the system exists. As a result of the above, the systems of this generation provide limited support for the correct representation of arguments. Contrary to the first generation, the second generation comprises systems where the identification of argumentation features is clear and noticeable. The emphasis is on the provision of knowledge management services, through the reuse of information and the employment of reasoning 
mechanisms (e.g. Hermes (Karacapilidis and Papadias, 2001), Belvedere (Suthers et al., 1995) and GeoMed (Karacapilidis et al., 1997)). As such, some of these systems are built to support learning activities (e.g. Reason!Able (van Gelder, 2003)). The third generation comprises systems that are deployed on the web and designed to support the collaborative production of arguments (e.g. PARMENIDES (http://cgi.csc.liv.ac.uk/ parmenides/), TruthMapping (http://www.truthmapping.com/), Cohere (http://cohere.open.ac.uk/) and CoPe_it! (Karacapilidis et al., 2009)). While they maintain the characteristics of those of the previous generation, they also provide mechanisms for administrating communities and allow the exploitation of existing resources through importing and exporting services.

Table 1. A classification of collaborative argumentation support systems

\begin{tabular}{|c|l|}
\hline $\begin{array}{c}\text { Argumentation } \\
\text { support systems }\end{array}$ & Characteristics \\
\hline $\begin{array}{c}\text { First generation } \\
\text { (1987-1995) }\end{array}$ & $\begin{array}{l}\text { Emphasis on visualization aspects. Adoption of argumentation aspects, without a clear } \\
\text { reference to specific model. Weak reuse of knowledge, no collaboration support. }\end{array}$ \\
\hline $\begin{array}{c}\text { Second generation } \\
\text { (1990-2007) }\end{array}$ & $\begin{array}{l}\text { Designed with regards to specific argumentation model. Appearance of mechanisms for } \\
\text { reasoning and decision making support. Web applications. Partial collaboration support. }\end{array}$ \\
\hline $\begin{array}{c}\text { Third generation } \\
\text { (2003-today) }\end{array}$ & $\begin{array}{l}\text { Designed for collaboration and convenient reuse of system and web resources. Early } \\
\text { provision for community support. }\end{array}$ \\
\hline
\end{tabular}

Despite the above developments, collaborative argumentation support systems continue to receive low user acceptance. As discussed in (Scheuer et al., 2010), the automatic analysis of single arguments with the intent to provide users with intelligent feedback enables active forms of support. Hence, it has strong potential in remedying the low acceptance of (and the low engagement of users with) existing argumentation support systems. In the majority of cases, this process concerns the construction of sound and syntactically valid arguments, which can then be analyzed to identify specific attributes that can be exploited by inference mechanisms, such as decision support algorithms. In line with the above, the approach proposed in the next section empowers argumentation support systems towards inducing the users' reasoning behavior.

\section{THE PROPOSED APPROACH}

An instance of an argumentation discourse exhibits a certain structure which allows one to draw conclusions about its state. For example, if one user is proposing an argument that receives low rating and at the same time there appear to be arguments attacking it with high rating, this means that the argument has most likely been defeated. Similar conclusions may be obtained by surveying the interrelation of arguments and not their content. Until now, these conclusions were left for the users of an argumentation support system, since the system was unable to capture and identify sequences of arguments that may express a certain attitude or behavior.

Our approach attempts to assess these structures by introducing the concept of Argumentation Sequence (AS), which can be simply described as "a set of arguments interrelated in a specific way". An example of different ASes is provided in Figure 2. The AS on the left may be interpreted as a sequence where the top argument is being under attack (only one argument supporting, which is under attack by two others), whereas the AS in the middle may indicate that the argument on top is being accepted (only one argument attacking, being under attack by two others). Similarly, the AS on the right may indicate that the root argument is of uncertain status. In fact, our approach aims at measuring the similarity among pairs of ASes. In order to achieve this, an innovative graph-based computational model has been introduced and described in detail in (Gkotsis and Karacapilidis, 2012). Our overall approach is based on the (commonly adopted in argumentative discourse and) following assumptions: 

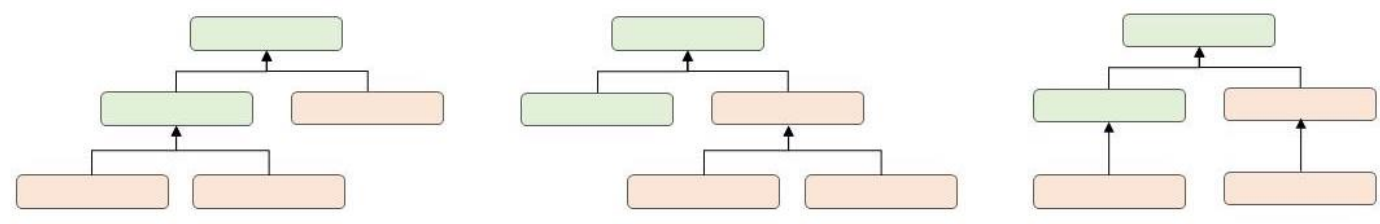

Figure 2. An example of three different Argumentation Sequences; while the size (5 arguments) and the depth of the sequences (until level 2) is the same, their interpretation in the argumentation context varies

- Similarly to the framework introduced by Dung (1995), the internal structure of arguments is not considered; each argument is an atomic entity.

- One or more attributes of the argument are exploited. An example of an attribute may be its rating, the name of the author or its publication date. The values of these attributes are used in order to introduce a fundamental distance function between different arguments (i.e. two arguments that have received exactly the same rating or belong to the same author have distance of value 0 ).

- Arguments are connected to each other in order to express explicitly (and exclusively) either agreement or disagreement.

- The discourse is structured and visualized as an argumentation tree. While there are cases where argumentation may be represented in other ways as well, the vast majority of the above systems adopt the tree-like representation of the information units (Freeman, 1991).

Our approach is influenced by similar graph and subgraph matching techniques but is tailored for the case of argumentation trees. The process of computing the similarity between two ASes includes the matching of vertices of one AS to those of the other by preserving their corresponding level. During this matching, we calculate the pairwise arguments' similarity for all levels. The matching must be such that the overall similarity measurement is maximized.
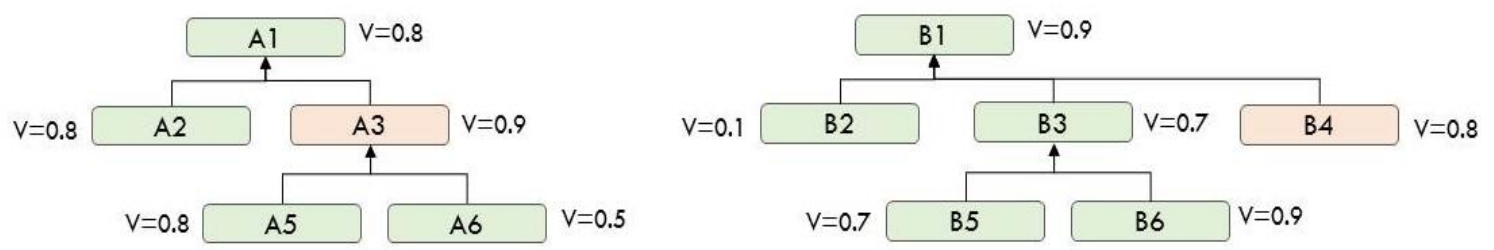

Figure 3. Two argumentation sequences with annotated rating

For instance, consider the two ASes shown in Figure 3. For each argument of the ASes, we presume that a rating submitted by the users exists, which is annotated. This rating is normalized on a scale from 0 to 1 . Our algorithm for computing the similarity of the ASes results in the following arguments-vertices matching: $\{\mathrm{A} 1, \mathrm{~B} 1\},\{\mathrm{A} 3, \mathrm{~B} 3\},\{\mathrm{A} 2, \mathrm{~B} 2\},\{\mathrm{A} 6, \mathrm{~B} 5\}$ and $\{\mathrm{A} 5, \mathrm{~B} 6\}$. The final similarity score, which is normalized on a scale from 0 to 1, is 0.72 . The partial similarity for the arguments of the second level (A2 and A3 versus B2, B3 and $\mathrm{B} 4$ ) is 0.28 . This low value is due to the fact that arguments A3 and B3 were selected for matching, even though they are significantly different (A3 is opposing its parent argument and B3 is supporting it, both with high rating). The reason for this high cost matching is because it returns high matching for the arguments of the third level (A4, A5, B5, B6), thus maximizing the overall score.

In order to further exploit the above similarity measurement, the Boolean property of equivalence between ASes is used in order to classify two ASes as argumentatively equivalent. Essentially, this property introduces a threshold value as the minimum value of matching across each and every level of the ASes. For the example described above, the low similarity value for arguments of level 2 would have returned that the ASes are not equivalent. This threshold value is specified by the user. Unless the partial similarity score for all levels is higher than the threshold value, the two ASes are not considered argumentatively equivalent. The benefits of the above similarity measurement and the consequent concept of equivalence are discussed below. 


\section{INDUCING REASONING BEHAVIOR}

Until now, we have argued about the need to reinforce argumentation models with intelligent processing mechanisms. For this reason, we have proposed the exploitation of argumentation sequences. We have also described intuitively (for details see (Gkotsis and Karacapilidis, 2012)) that it is possible to easily identify equivalent argumentation sequences. This section proposes specific cases where the adoption of the proposed approach may assist in Human Behavior Understanding (HBU), as defined in (Salah et al., 2010). As reported in the above work, HBU refers to "advanced pattern recognition techniques to automatically interpret complex behavioral patterns generated when humans interact with machines or with others". In this section, we sketch representative cases where our approach may be used to capture meaningful aspects of the users' behavior and consequently improve their collaboration by augmenting the awareness offered by an argumentation support system.

The first set of cases assumes that a predefined, meaningful set of argumentation sequences exists. The aim is to identify other sequences equivalent to the above ("situated" sequences). The predefined sequences may initially be system defined, but every user might choose to manually label more, or even exclude some. For instance, it is possible to include the ASes of Figure 2 and label them accordingly. More generally, a set of ASes that accounts the arguments' rating may be populated and labeled as "accepted arguments" and "defeated arguments". The results in favor of HBU may be one or more of the following:

- Fight selective exposure. Users are naturally inclined to ignore arguments or ideas they have already devalued. As reported in (Jonas et al., 2001), after making a decision, people prefer supporting instead of conflicting information. This bias may undermine the ability of users to identify weak points in their course of thinking. Spotting defeated arguments in a neutral, mechanical way will increase awareness for the users and will reduce the above bias. The result might be either the individual or the collective change of strategy regarding a debatable subject.

- Similarly to the above, automatic identification of interesting argumentation patterns may reduce confirmation bias. As reported in (Kuhn, 1991), people might overlook counter-arguments and may overvalue their initial predictions or estimations. Matching ASes against other labeled patterns of argumentation assists in bringing to surface parts of the discourse in an objective way and recommends reinstating specific arguments.

- More generally, the exploitation of arguments' rating may highlight information that the users overlook when examining the discourse. It will allow for increased introspection in the experienced users and will enable the more efficient survey, reconstruction and understanding of the argumentative discourse, especially for the newcomers. Administrators may be able to construct rich queries and efficiently search the discussions. Moreover, the above activities may be facilitated through notification mechanisms that are triggered every time an argumentation sequence matches a desired pattern.

The above cases are based on the existence of a collection of predefined, labeled ASes and allow the automatic identification of equivalent ASes as they are formed. Apart from the above, we believe that ASes may be subject to further exploitation through the mining and automatic discovery of emerging, otherwise unnoticeable patterns of communication. In order to achieve this, instead of the rating attribute, we consider the author or the publication date attributes. In this way, when a specific communication pattern based on users' identity reoccurs, the system will be able to report this information. For instance, if an attack relationship between arguments is repeating in a short period between two or more users, this behavior will be identified, without the need to exploit the rating attribute. More detailed ways on what kinds of unnoticed behaviors may be discovered are as follows:

- Users might be engaging in an endless, turn-based debate. In that case, the proposed approach will observe that a series of arguments by the same authors appears as ASes in the system, which will be recognized as an endless debate. In this case, the primary attribute to be exploited would be the author names. However, it is also possible to induce the same behavior by examining the time difference between different posts of arguments (i.e. short time interval between attacking arguments). In both cases, this user engagement will be spotted automatically. 
- An a posteriori analysis of the discourse might return unnoticed patterns of behavior within the community. For example, it might be the case that some users might be colluding in order to mislead the discussion or repeatedly attack another user. In that case, several ASes containing the same group of people and replicating the same, aggressive behavior are discovered.

- Similarly to the above, a common problem in massive, public deliberation is the inflammation of the discourse with aggressive content and behavior. In this direction, general communication platforms, such as fora and other content management systems, may take measures against these users through banning or deleting their account. However, in the majority of the cases, these actions are either carried out manually or are based on simple rules. Thus, they are of limited application in an argumentation context. In our approach, the automatic identification of a specific behavior can trigger measures against this attitude.

Furthermore, it is worth noting that our approach can be particularly useful in specific applications of argumentation. The most common use of argumentation is in supporting learning. An argumentation support system powered by our model will help learners understand their behavior. Through the exploitation of ASes, the tutor is able to spot repeating reasoning behaviors. Similarly, learners are able to understand better how to confront argumentation by noticing the emerging interrelations of their contributions and ultimately improve their reasoning skills.

Another application of argumentation is found in discourse analysis and decision making support within organizations. For example, argumentation support systems such as Compendium (http://compendium.open.ac.uk/), which conforms to the IBIS approach, are built with the aim to capture organizational knowledge and complies with the principles of argumentation. A common case is when the same arguments may surface in different discussions and require further analysis. Our approach may be tailored in order to support the exploitation of existing resources with automated identification mechanisms.

The cases described above provide concrete examples of how a typical argumentation model may be further extended in order to uncover patterns of reasoning behavior. In all these cases, the main intention is to reveal and reduce behaviors related to user biases in rational and critical thinking. To achieve this, the complex but semantically rich structure of the Argumentation Sequence can be the central unit of the proposed computational model. Even though our approach adopts general concepts of IBIS like systems, it is worth discussing its limitations. First of all, the ASes must follow a tree structure, which is a common practice (as discussed earlier). Secondly, the assessment of argumentation structures can identify certain behavior, but is far from appearing as a semantically complete approach. While the exploitation of the proposed model accomplishes the annotation of some parts of an argumentative dialogue and highlights similarities with respect to previous discussions or predefined patterns, it cannot manage every case. Therefore, our approach can be viewed as a complementary, semi-automatic tool that adds awareness features in a broad category of argumentation support systems. Compared to related approaches, Belvedere's advisor (Paolucci et al., 1996) automatically assesses the validity of components of arguments either through its pairwise comparison of relations against an expert's diagram (consistency advisor), or through the rule-based, syntactic validation of diagrams against explicit conditions. The DOCE algorithm follows a machine learning approach in order to classify arbitrarily sized clusters of contributions (Mclaren et al., 2010). Our approach shares the same goals with DOCE, but introduces a more refined algorithm that manages to capture and discern structures, which would have been clustered equally in DOCE.

\section{CONCLUSIONS}

Argumentation is a form of informal reasoning. Even though it is supposed to be governed by rationalization, it remains an inherently complex process where humans often behave irrationally and unexpectedly. We have proposed an approach for assessing complex argumentation structures in order to identify meaningful reasoning patterns and accordingly induce the reasoning behavior of argumentation support systems' users. Our work does not intend to propose new ways of structuring and validating an argumentative discourse; instead, it aims at studying how to extend and exploit a widely adopted argumentation model (i.e. IBIS) for understanding and inducing human behavior. Through the provision of various cases, this paper is primarily arguing why such an approach is sensible and may contribute to both theoretical and practical aspects of argumentation. 


\section{ACKNOWLEDGMENT}

The work presented in this paper is supported by the OpenBio-C project (www.openbio.eu), which is co-financed by the European Union and Greek national funds through the Operational Program Competitiveness, Entrepreneurship and Innovation, under the call RESEARCH - CREATE - INNOVATE (Project id: T1EDK- 05275).

\section{REFERENCES}

Conklin, J. and Begeman, M.L., 1987. gIBIS: A hypertext tool for team design deliberation. Proceedings of the ACM Conference on Hypertext, pp. 247-251, New York, NY, USA.

Dung, P.M., 1995. On the acceptability of arguments and its fundamental role in non-monotonic reasoning, logic programming and n-person games. Artificial Intelligence, Vol. 77, No. 2, pp. 321-357.

Freeman, J., 1991. Dialectics and the macrostructure of arguments: a theory of argument structure. Walter de Gruyter, Berlin/New York.

Gkotsis, G. and Karacapilidis, N. (2012). A computational model for the identification and assessment of structural similarities in argumentative discourses. Journal of Intelligent Information Systems, Vol. 39, No. 3, pp. 789-811.

Jonas, E. et al., 2001. Confirmation bias in sequential information search after preliminary decisions: An expansion of dissonance theoretical research on selective exposure to information. Journal of Personality and Social Psychology, Vol. 80, No. 4, pp. 557-571.

Karacapilidis, N. and Papadias, D., 2001. Computer supported argumentation and collaborative decision making: the Hermes system. Information systems, Vol. 26, No. 4, pp. 259-277.

Karacapilidis, N. et al., 2009. Tackling cognitively-complex collaboration with CoPe_it!. International Journal of Web-Based Learning and Teaching Technologies, Vol. 4, No. 3, pp. 22-38.

Karacapilidis, N. et al., 1997. Collaborative Environmental Planning with GeoMed. European Journal of Operational Research, Vol. 102, No. 2, pp. 335-346.

Kuhn, D., 1991. The skills of argument. Cambridge University Press, Cambridge, UK.

Kunz, W. and Rittel, H., 1970. Issues as Elements of Information Systems, Working Paper No. 131, Studiengruppe für Systemforschung, Heidelberg, Germany.

Mclaren, B.M. et al., 2010. Supporting collaborative learning and e-discussions using artificial intelligence techniques. International Journal of Artificial Intelligence in Education, Vol. 20, No. 1, pp. 1-46.

Paolucci, M. et al., 1996. Automated advice-giving strategies for scientific inquiry. Proceedings of the Third International Conference on Intelligent Tutoring Systems, Vol. 1086, pp. 372-381.

Salah, A. et al., 2010. Challenges of human behavior understanding. In: Salah, A.A. et al. (eds.) Human Behavior Understanding, LNCS, Vol. 6219, pp. 1-12, Springer, Heidelberg.

Scheuer, O. et al., 2010. Computer-supported argumentation: A review of the state of the art. International Journal of Computer-Supported Collaborative Learning, Vol. 5, No. 1, pp. 43-102.

Suthers, D. et al., 1995. Belvedere: Engaging students in critical discussion of science and public policy issues. Proceedings of the 7th World Conference on Artificial Intelligence in Education, Washington D.C, USA, pp. 266-273.

van Gelder, T.J., 2003. Enhancing deliberation through computer supported argument mapping. In: Kirschner, P.A. et al. (Eds.), Visualizing argumentation: Software tools for collaborative and educational sense-making, pp. 97-115, Springer-Verlag, London. 